\title{
SIMULATION EFFECTS OF SURFACE GEOMETRY AND WATER OPTICAL PROPERTIES ON HYDROGRAPHIC LIDAR RETURNS
}

\author{
Song Yang ${ }^{\star 1,2,3,4}$, Qian Sun ${ }^{1,2,3,4}$,Yongchao Zheng ${ }^{1,2}$ \\ ${ }^{1}$ Beijing Institute of Space Mechanics \& Electricity, Beijing 100094, China \\ ${ }^{2}$ Key Key Laboratory for Space Laser Information Perception Technology of CAST, \\ ${ }^{3}$ Beijing Key Laboratory of Advanced Optical Remote Sensing Technology, Beijing 100094, China \\ ${ }^{4}$ Key Laboratory for Optical Remote Sensor Technology of CAST, China \\ *Email:kellyyszx@163.com
}

\begin{abstract}
. Water LiDAR model was applied to simulate the returned waveforms of hydrographic LiDAR considering the effects of surface geometry and water optical properties. The signal to noise ratio(SNR) of bottom returned peak was considered as a criterion for performance of hydrographic LiDAR. The behavior of LiDAR was sensitive to water optical properties and it was insensitive to water surface roughness.
\end{abstract}

\section{INTRODUCTION}

Water surface altimetry and bathymetry are important factors for geomorphology, hydrology ${ }^{[1]}$, and meteorology. Water surface altimetry and bathymetry can be measured with several methods by using sonar, radar, and optical imaging. However, these techniques have some difficulties when used on swallow water, such as: 1) cannot achieve full spatial coverage on coastal water (sonar), 2) the lack of high depth accuracy due to the low penetration of radar and optical imaging.

Airborne hydrographic LiDAR has been considered as a suitable sensor for bathymetry because of its low latency, high accuracy and high density data. Take advantages of the blue-green visible wavelength window, blue-green laser has a good performance in long distance underwater sensing, especially the subsurface sensing. Thus the airborne hydrographic LiDAR can get the returned signals from the water surface ${ }^{[1]}$, water column $^{[2]}$ and water bottom ${ }^{[3,4]}$ for altimetry and bathymetry.

Due to the scattering and absorption property of water, the effects of energy loss and direction changing can be generated for the optical beams. These effects may introduce the pulse spreading and pulse stretching, which play important roles in beam propagation. Several Mathematical models $\mathrm{s}^{[1,3,4]}$ are developed to simulate the returned signals from the hydrographic Lidar in order to optimize parameters of lidar systems under development or to estimate a system efficiency in a given conditions.

In this paper, we take water LiDAR simulation model to simulate the returned waveforms for airborne LiDAR with 532nm frequency. The SNR of simulated waveforms are taken to evaluate the behavior of LiDAR in different conditions. The effects of water surface slopes and water optical properties are also discussed.

\section{METHODOLOGY}

\subsection{Water LiDAR Model}

The Water LiDAR model ${ }^{[5]}$ presented by $\mathrm{H}$. Abdallah et.al., is a generalized model for LiDAR waveforms with wavelength in the optical spectrum domain from $300 \mathrm{~nm}$ to $1500 \mathrm{~nm}$. This model has good features including: 1) description of the water surface geometry based on bidirectional reflectance distribution function $(\mathrm{BRDF})^{[6]}$, 2) description of the optical property of water column for different wave length based on the laws of radiative transfer, 3) calculation of SNR which considers detector noise and background solar noise.

The returned waveforms from hydrographic LiDAR system can be written as ${ }^{[4,5]}$

$P_{T}(t)=P_{s}(t)+P_{c}(t)+P_{b}(t)+P_{b g}(t)+P_{N}(t)$

where $P_{T}(t)$ is the total power received, $P_{s}(t)$ is the power returned from the surface, $P_{c}(t)$ is the power returned from the column, $P_{b}(t)$ is the power returned 
from water bottom, $P_{b g}(t)$ is the background solar radiation power, $P_{N}(t)$ is the detector noise power, and $t$ is the time scale.

\subsection{Signal to Noise Ratio}

The SNR of the hydrographic LiDAR is shown as:

$$
S N R=\frac{P_{s}(t)+P_{c}(t)+P_{b}(t)}{\sigma_{b g}(t)+\sigma_{N}(t)}
$$

$\sigma_{b g}(t)$ is the standard deviation of the background solar radiation power, and $\sigma_{N}(t)$ is the detector noise power.

\subsection{Effect of Surface Geometry}

The returned power from the surface is given by

$$
P_{s}(t)=\omega_{s}(t) * P_{s},
$$

with $\omega_{s}(t)$ is the returned waveform of the pulse propagation in a perfect and lossless channel from the water surface, and $P_{s}$ is the instant echoes from the water surface ${ }^{[3,5]}$,

$$
P_{s}=P_{e} T_{a t m}^{2} A_{R} \eta_{e} \eta_{R} \frac{f}{(H / \cos \theta)^{2}} .
$$

Here $P_{e}$ is the emitted power of the LiDAR system, $T_{\text {atm }}$ is the loss due to the atmosphere, $A_{R}$ is the receiver area, $H$ is the sensor altitude, $\theta$ is the local incidence angle, $\eta_{e}$ and $\eta_{R}$ are the optical efficiency of the emitter and receiver. $f$ is the loss factor from the surface and is based on BRDF of the water surface. Here we use the geometric model of Cook and Torrance to describe the behavior of surface geometry. The equation of loss factor $f$ is ${ }^{[6]}$

$$
f=\frac{D O F_{r}}{4 \cos ^{2} \theta},
$$

where

$$
D=\pi^{-1} r^{-2} \cos ^{-4} \theta \exp \left(-(\tan \theta / r)^{2}\right)
$$

is the microfact distribution function ${ }^{[6,7]}, \mathrm{O}$ is the geometric attenuation factor of BRDF, and $F_{r}$ is the Fresnel reflection of light on the water surface.

\subsection{Effect of Optical properties of water}

The optical properties of water can be described by absorption coefficient $a(\lambda)$, scattering coefficient $b(\lambda)$, attenuation coefficient $c(\lambda)$, scattering function $\beta(\varphi)$, and diffuse attenuation coefficient $k$. The empirical relationship between diffuse attenuation and optical properties ${ }^{[1]}$ is established as

$$
\begin{aligned}
& k=c(\lambda)[0.19 \times(1-b(\lambda) / c(\lambda))]^{\frac{b(\lambda)}{2 c(\lambda)}} \\
& \text { where }^{[8]} c(\lambda)=a(\lambda)+b(\lambda), \\
& a(\lambda)=a_{w}(\lambda)+a_{y}(\lambda)+a_{p h}(\lambda)+a_{s}(\lambda), \\
& b(\lambda)=b_{w}(\lambda)+b_{p h}(\lambda)+b_{s}(\lambda) .
\end{aligned}
$$

Here the subscripts ' $w$ ', ' $y$ ', ' $p h$ ', 's' represent pure water, yellow substances, phytoplankton and sediment, respectively.

The returned waveform from the water column and bottom is calculated as

$$
\begin{aligned}
& P_{c / b}=P_{e} T_{a t m}^{2} A_{R} \eta_{e} \eta_{R} \frac{(1-f)^{2} \exp (-2 k z / \cos \theta)}{(n H+z / \cos \theta)^{2}} \\
& \cdot\left(\operatorname{sgn}\left(\frac{z-Z}{Z}\right) \beta(\varphi)+\left(1-\operatorname{sgn}\left(\frac{z-Z}{Z}\right)\right) \frac{R_{b}}{\pi}\right)
\end{aligned}
$$

where $n$ is the water refractive index, $R_{b}$ is the bottom albedo, $z$ is the depth underwater, $Z$ is the depth of the bottom, and $\operatorname{sgn}(\cdot)$ is the step function.

\section{RESULTS}

\subsection{Simulation results for hydrographic LiDAR}

According to water LiDAR model, the returned waveforms from traditional hydrographic LiDAR are shown in Fig.1. The relevant parameters are shown in Table. 1.

Table.1 Parameters used in simulation

\begin{tabular}{|c|c|c|c|}
\hline Parameters & LIDAR & Parameters & LIDAR \\
\hline$\lambda(\mathrm{nm})$ & 532 & $\eta_{R}$ & 0.5 \\
\hline$H(\mathrm{~m})$ & 400 & $T_{\text {atm }}$ & 0.9 \\
\hline$P_{e}(\mathrm{MW})$ & 1 & $\beta(\varphi)\left(m^{-1} s r^{-1}\right)$ & 0.0014 \\
\hline$\theta\left(^{\circ}\right)$ & 20 & $r$ & 0.1 \\
\hline
\end{tabular}




\begin{tabular}{|c|c|c|c|}
\hline$A_{R}\left(\mathrm{~m}^{2}\right)$ & 0.025 & $n$ & 1.33 \\
\hline$\eta_{e}$ & 0.9 & $R_{b}$ & 0.1 \\
\hline
\end{tabular}
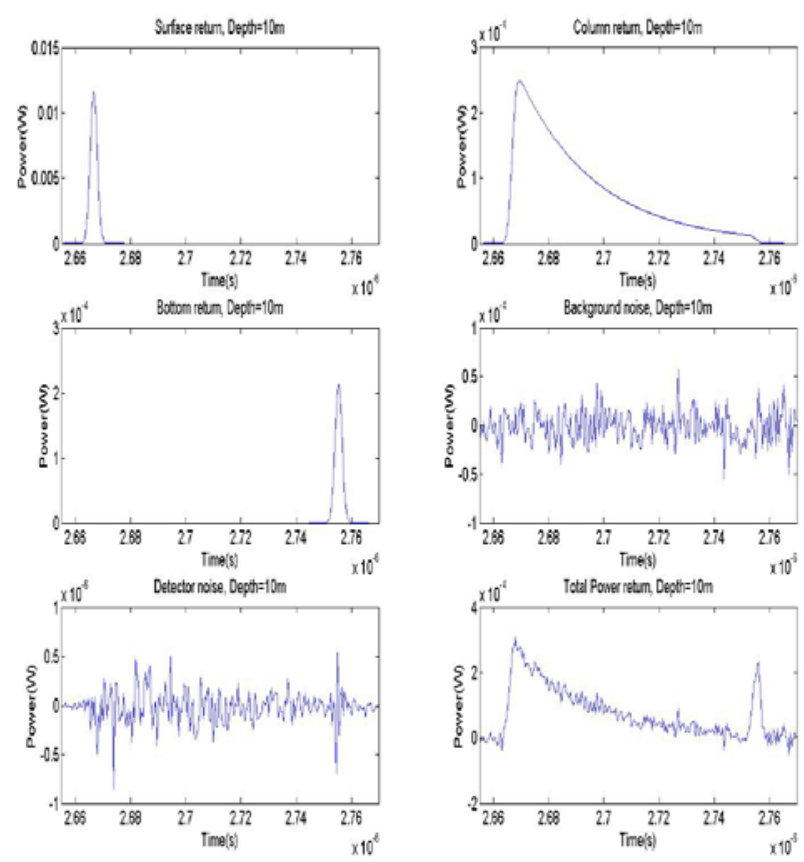

Fig.1 Simulated returned waveforms of hydrographic LiDAR with wavelength $532 \mathrm{~nm}$. The water is of Case II quality (the average concentration of phytoplankton $C_{\mathrm{ph}}=4 \mathrm{mg} / \mathrm{m}^{3}$, sediment $S=2.6 \mathrm{mg} / 1$ )

\subsection{SNR for different depth}

The SNR of bottom returned waveforms at different depth is discussed in this section. Fig.2 shows SNR distribution of peaks of the bottom returned waveforms. The results shows that SNR is linearly decreases with water depth.

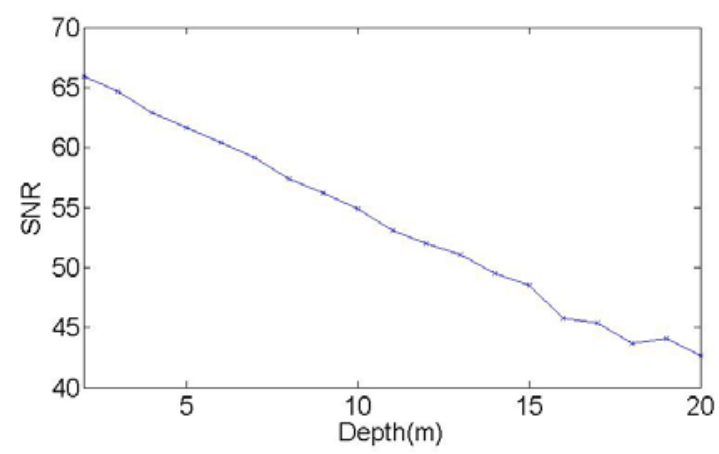

Fig.2 SNR of the bottom returned waveform vs. water depth

\subsection{Effect of Surface Geometry}

The effect of surface geometry is mainly relevant with the surface slope parameter $r$. Small values of $r$ represent gentle facet slopes and behaves as a specular facet, while large values of $r$ represent steep facet slopes and behaves as a diffuse facet. The performance of SNR of bottom returned waveforms versus surface slope parameter $r$ is exhibited in Fig.3. When $r$ was small( $(r<0.1)$, SNR of bottom waveform was low since the returned light is highly directional and has low overlap with receiver area. When $r$ was large $(r>0.1)$, SNR became stable since the returned light is unidirectional and get insensitive with incident angle.

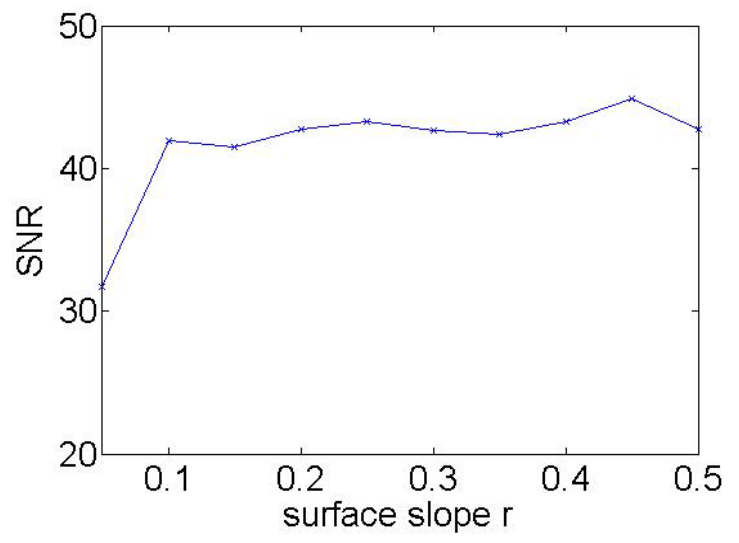

Fig.3 SNR of the bottom returned waveform vs. surface slope

\subsection{Effect of Optical properties of water}

In Section 2.4, the relationship between optical properties of water and diffuse attenuation coefficient $k$ is shown in Eq.(7). We compare the total power return of Case I water and Case II water. The scattering and attenuation effects for Case II water are more serious than that for Case I water.

Table 2. Comparisons of two sites with different optical properties

\begin{tabular}{|c|c|c|}
\hline Parameters & Case I & Case II \\
\hline$k\left(\mathrm{~m}^{-1}\right)$ & 0.0449 & 0.1572 \\
\hline$c\left(\mathrm{~m}^{-1}\right)$ & 0.0731 & 0.4912 \\
\hline$a\left(\mathrm{~m}^{-1}\right)$ & 0.0412 & 0.1233 \\
\hline$b\left(\mathrm{~m}^{-1}\right)$ & 0.0319 & 0.3679 \\
\hline
\end{tabular}



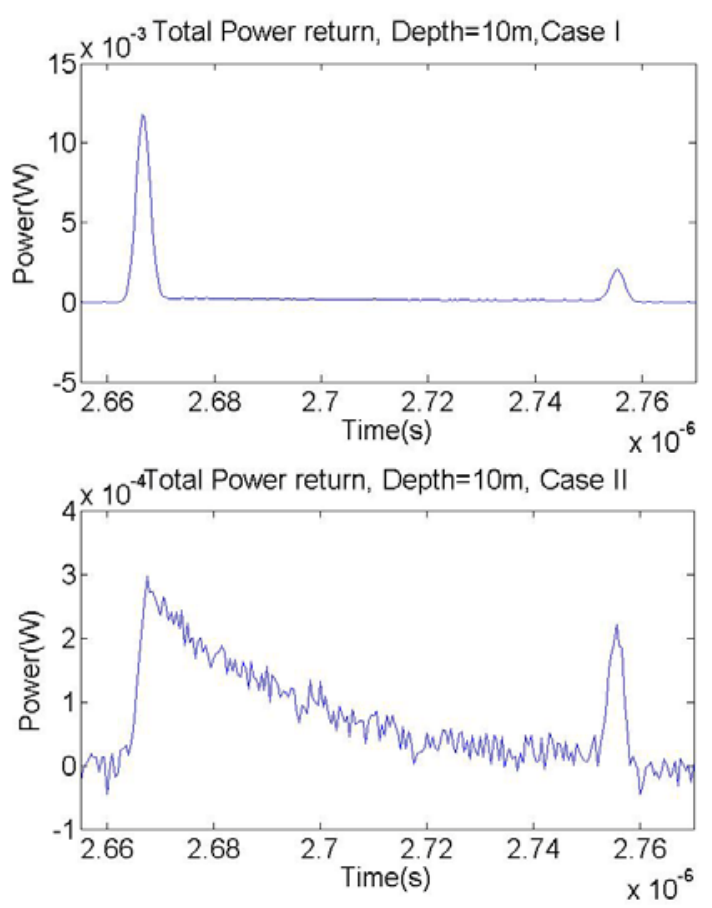

Fig.4 Comparison of total power return in Case I water and Case II water. The water depth is $10 \mathrm{~m}$.

\section{CONCLUSION}

Water LiDAR simulation model was applied to describe the returned waveforms of airborne hydrographic Lidar. The SNR of peaks of bottom returned waveforms are analyzed and SNR is linearly decreases with depth. The Effect of Surface Geometry is also considered in this paper, and the SNR is stable with the water surface slope $r \in[0.1,0.5]$. The performance of hydrographic Lidar is very sensitive to optical properties of water, and prior information of water optical properties is significant for estimation the maximum depth of hydrographic Lidar.

\section{ACKNOWLEDGEMENTS}

This work was supported by National Natural Science Foundation of China(11574400 , 11204379, 11304013, 61705007), GuangXi innovative Development Grand Grant under Grant No. GuiKe AA 18118038, CAST Foundation for Distinguished Young Scholars(2019).
[1] G. C. Guenther, presented at the NOAA Professional Paper Ser. NOS1, Rockville, MD, 1985.

[2]A. Collin, P. Archambault, and B. Long, IEEE Trans. Geosci. Remote Sens., vol. 46, no. 10, pp. 2947-2955, Oct. 2010.

[3] J. Feigels, Ocean Opt. XI, vol. 1750, pp. 473-484, 1992.

[4] H. M. Tulldahl and K. O. Steinvall, Appl. Opt., vol. 42, no. 12, pp. 2462-2483, Apr. 2004.

[5] Hani Abdallah, Nicolas Baghdadi, et.al. IEEE Trans. Geosci. Remote Sens., Vol. 9, no. 4, pp.744-748, July.2012.

[6]R. L. Cook and K. E. Torrance, ACM Trans. Graph., vol. 1, no. 1, pp. 7-24, Jan. 1982.

[7]P. Beckmann and A. Spizzochino, The Scatter of Electromagnetic Waves From Rough Surfaces. Norwood, MA: Artech House, 1987.

[8] C. D. Mobley, Light and Water: Radiative Transfer in Natural Waters.San Diego, CA: Academic, 1994.

\section{REFERENCES}

